DOI: $10.26851 /$ RUCP.27.5

\title{
SOBRE EL “NUEVO CONSTITUCIONALISMO LATINOAMERICANO” *
}

\author{
The "New Latin American Constitutionalism"
}

\section{Roberto Gargarella ${ }^{1}$}

\begin{abstract}
Resumen: Este artículo estudia la evolución del constitucionalismo latinoamericano desde sus orígenes, prestando particular atención a la última oleada de reformas constitucionales que tuvo lugar hacia finales del siglo XX y que diera lugar al llamado "nuevo constitucionalismo latinoamericano". El texto llama la atención sobre las profundas continuidades existentes entre este "nuevo" constitucionalismo y el más tradicional y arcaico - "viejo"- constitucionalismo regional, tanto en lo relativo a la organización de los derechos, como en lo vinculado con el diseño institucional predominante. El artículo sostiene, en particular, que la organización del poder que todavía hoy predomina en una mayoría de constituciones de la región sigue estando definida por los rasgos elitistas y autoritarios propios del momento liberal-conservador que rigiera en América Latina entre los años 1850 y 1890.
\end{abstract}

Palabras clave: América Latina, derechos, democracia, "sala de máquina", Constitución.

Summary: This article studies the evolution of Latin American constitutionalism from its origins, paying particular attention to the last wave of constitutional reforms that took place towards the end of the 20th century and that gave rise to the so-called "new Latin American constitutionalism". The text draws attention to the deep continuities between this "new" constitutionalism and the more traditional and archaic - "old"- regional constitutionalism, regarding the organization of rights, as well as the predominant institutional design. The article argues, in particular, that the organization of power that still predominates in a majority of constitutions in the region continues to be defined by the elitist and authoritarian features of the liberal-conservative period that prevailed in Latin America between the years 1850 and 1890.

Key words: Latin America, rights, democracy, "engine room”, Constitution.

\footnotetext{
* Artículo recibido el 30/11/2017 y aceptado el 19/03/2018.

1 Universidad de Buenos Aires. Universidad Torcuato Di Tella. Correo electrónico: roberto.gargarella@ gmail.com
} 


\section{Introducción}

A partir de la aprobación de las Constituciones de Colombia (1991); Venezuela (1999); Ecuador (2008) y Bolivia (2009), se suele hablar de la existencia de un "nuevo constitucionalismo latinoamericano". Sin embargo, me interesará decir aquí que el llamado "nuevo constitucionalismo" es demasiado parecido a lo que ya teníamos.

En mi examen de las diferentes constituciones de la región, asumiré siempre que ellas -más allá de las obvias complejidades que les son propias-, se encuentran unidas por ciertos rasgos comunes. Ante todo, considero que todas ellas aparecen divididas en dos partes principales, esto es, la sección orgánica, referida a la organización del poder y la sección dogmática, referida a la declaración de derechos. La primera de tales partes define los rasgos principales de lo que podríamos llamar la "maquinaria democrática" (ella establece, por ejemplo, los modos en que van a funcionar y relacionarse las distintas ramas del Poder, típicamente, el Poder Ejecutivo, el Legislativo y el Judicial). La segunda parte, mientras tanto, determina cuáles son los derechos fundamentales que cada una de las comunidades involucradas reconoce como tales.

A continuación, entonces, daré cuenta de los modos en que ha ido evolucionando el constitucionalismo latinoamericano en todo este tiempo y prestaré atención a lo que ha permanecido y cambiado en el mismo. Ello así, para estar en mejores condiciones de evaluar el legado que recibimos hoy, dos siglos después de su inicio.

\section{Cuatro etapas del constitucionalismo latinoamericano}

La evolución del constitucionalismo regional podría ser dividida en cuatro etapas, dirigidas: i) el constitucionalismo "experimental", particularmente preocupado por la consolidación de la independencia (1810-1850); ii) el "fundacional", interesado, sobre todo, en la consolidación económica (1850-1917); iii) el "social", orientado a recuperar la olvidada "cuestión social" (1917-1980) y; iv) el de los derechos humanos, dirigido a sanear la crisis de derechos humanos propia de la década del 1970 (1980-2000).

De esas cuatro etapas principales, aquí me ocuparé fundamentalmente de la segunda y la tercera. Entiendo que solo estas dos etapas resultan determinantes para la vida del constitucionalismo regional, en virtud del impacto que tuvieron sobre la estructura de las constituciones latinoamericanas. Según diré, las reformas introducidas a mediados del siglo XIX fueron decisivas en relación con la estructura de poderes de la Constitución: ellas definieron el carácter de dichas estructuras de poderes, haciendo que éstas sean lo que todavía hoy son. Mientras tanto, las reformas introducidas en la primera mitad del siglo XX resultaron decisivas en relación con las declaraciones de derechos propias de las Constituciones latinoamericanas: ellas definieron el carácter de tales declaraciones de derechos que todavía hoy se encuentran moldeadas conforme a los rasgos que se le impusieron entonces.

A continuación, enumeraré los dos momentos fundamentales del constitucionalismo regional, para ir centrándome, a partir de allí, en el aspecto normativo que más interesa a este artículo. 


\subsection{Un sistema de "frenos y contrapesos" desbalanceado hacia el Ejecutivo: la primera "marca de identidad" del constitucionalismo regional}

A mediados del siglo XIX se produjo en América Latina un movimiento político crucial, a partir del paulatino pero decidido acercamiento entre las fuerzas liberales y conservadores que, hasta ese momento, y en una mayoría de países, se habían enfrentado en combates sangrientos. En algunos países como Argentina, Brasil o México, dicho acercamiento se manifestó con las fuerzas opositoras negociando juntas, sentadas en torno a la misma mesa de discusión constitucional. En Chile, el pacto adquirió formas "diferidas", cuando el "duro" conservadurismo constitucional consagrado por la Constitución de 1833 comenzó a "deshelarse", a partir de su gradual liberalización. En Colombia, en cambio, el proceso siguió el camino inverso al chileno, cuando el "duro" liberalismo constitucional impuesto por las sucesivas constituciones de 1853, 1858 y 1863, comenzó a perder fuerza para abrir paso a su gradual conservadurización, que se consolidara en la Constitución de 1886 (Barros Arana, 1913; Basadre, 1949; Bushnell, 1993; Jaramillo Uribe, 1964; Molina, 1973).

Este período es especialmente importante, por un lado, porque fue allí donde se dictaron muchas de las Constituciones más trascendentes de la historia latinoamericana. De hecho, muchos de los textos aprobados entonces (los de Argentina en 1853, Colombia en 1886, México en 1857, entre otros), siguen siendo puntos de referencia ineludibles para entender el actual constitucionalismo de la región. En particular, sostuve ya que fue entonces cuando tomó su forma (en buena medida) definitiva la estructura de organización de poderes que sigue distinguiendo hoy a una mayoría de las Constituciones de la región. Para sostener lo anterior, conviene reconocer que en el período previo -el post-independentista-, la disputa constitucional tendía a enfrentar a liberales y conservadores que se alternaban en el poder y buscaban imponer, unos sobre los otros, sus propios proyectos constitucionales. La Constitución conservadora de Chile de 1823, fue sucedida por la más liberal de 1828, que fue reemplazada por la más conservadora de 1833; el constitucionalismo conservador colombiano de 1843 fue sucedido por el ultra-liberal desde 1853; el liberalismo de la Constitución mexicana de 1824 fue seguido por el dictado de las Leyes Constitucionales de 1836 y las Bases Orgánicas de 1843, que reaccionaron frente a aquella; y así.

Esta sucesión violenta de gobiernos, políticas y constituciones se tradujo en ordenamientos constitucionales que oscilaron fuertemente entre extremos (típicamente, de las fórmulas del poder concentrado en nombre del orden, a las "respuestas del miedo" y la búsqueda de la limitación del poder) dentro de moldes muy variados y abiertos a la experimentación (Blanco Acevedo, 1939; Bazant, 1977; Negretto y Rivera, 2000; Valencia Villa, 1987). En cambio, desde la consolidación del pacto liberal-conservador, buena parte de esa experimentación inicial se terminó: se acabaron las búsquedas decididamente corporatistas; decayeron las exploraciones monárquicas; se terminaron los Ejecutivos de "tres cabezas"; desaparecieron del mapa del constitucionalismo instituciones como el "Poder Moral", el "Poder Moderador" o los "Senadores Visitadores" (Halperín Donghi, 1980; Jaksic, 2001; Gargarella, 2010). 
Desde mediados del siglo XIX comenzamos a encontrarnos con esquemas constitucionales más definidos, menos abiertos a la experimentación, y que desde entonces admitieron sólo unas pocas variaciones dentro de márgenes más bien estrechos. La estructura general del poder se estabilizó así en los rasgos definidos por el pacto liberal-conservador: un esquema de "frenos y contrapesos" o "balances y contra-balances" finalmente "desbalanceado" hacia el Ejecutivo, que implicaba -un producto del pacto- una parcial superposición entre las demandas de los liberales (un sistema de checks and balances al estilo norteamericano) y los conservadores (un sistema organizado en torno al Poder Ejecutivo).

Algo similar podía verse en materia de organización territorial (en la tensión centralismo-federalismo) y, muy especialmente también, en materia de derechos. La pregunta era (al menos, en la gran mayoría de los casos) cómo integrar las demandas de liberales y conservadores, en un área en donde las diferencias entre ellos eran particularmente significativas, debido a la presencia de la cuestión religiosa por la cual habían recurrido a las armas durante décadas. Las fórmulas de integración por las que optaron entonces fueron diversas, y dejaron mucho que desear en una mayoría de casos. Ellas incluyeron desde el silencio en la materia, por el que se inclinaron los constituyentes mexicanos de 1857 (omitir las demandas de ambos grupos) a la doble invocación y directa superposición de ambas demandas, por las que se inclinaron los constituyentes argentinos de 1853 (tolerancia de cultos en el artículo 14 y status especial para la religión católica en el artículo 2).

Sobre lo anterior podría decirse, en primer lugar, que resulta curiosa fórmula de organización del poder por la que optaron los artífices del pacto liberal-conservador: un modelo de "frenos y contrapesos" finalmente "desbalanceado". El punto es interesante porque el esquema escogido implicó abrir una herida profunda, y desde un comienzo, a un modelo -el de los checks and balances- que tenía como principio organizador, justamente, al del equilibrio. Alguien puede encontrar muchas razones para objetar el esquema de organización del poder propuesto en su momento por James Madison, pero, en todo caso, el mismo ofrecía como virtud distintiva el equilibrio de poderes, basado en el igual poder relativo que se asignaba a cada una de las distintas ramas del poder. $\mathrm{Al}$ romper ese equilibrio, desbalanceando a la organización de poderes en dirección al Ejecutivo (esto es, lo que proponía Alberdi, al señalar la necesidad de combinar al modelo norteamericano con el modelo chileno), toda la lógica del sistema de checks and balances -que le daba sentido y atractivo- resultaba, desde el primer instante, puesta en directo riesgo. Muchos de los sobrevivientes problemas, que afectaron a la vida institucional latinoamericana tienen que ver con esa dudosa, difícilmente justificable, opción inicial (Alberdi, 1920; 1981; Loveman, 1993; Negretto y Rivera, 2000).

Destacaría, además, que el acuerdo liberal-conservador refiere también a la presencia de un "tercero excluido", esto es, las fuerzas radical-republicanas que habían ejercido una importante presión dentro de la discusión constitucional desde comienzos del siglo XIX. Según veremos, esta fundamental exclusión, que tendería a ser parcialmente reparada en el siglo XX, resulta determinante para explicar el tipo de fórmulas constitucionales finalmente aprobadas en el período fundacional. En otros 
términos, las Constituciones entonces moldeadas pueden entenderse tanto a partir de las fuerzas que le dieron forma, como a partir de las iniciativas que resultaron dejadas de lado: las que proponían -digámoslo así- "derechos políticos ampliados y derechos de propiedad limitados"; gobiernos más descentralizados y democráticos; un énfasis marcado sobre la "cuestión social" (Gargarella, 2010; 2013).

\subsection{Declaraciones de derechos (sociales, económicos y culturales) robustas: la segunda "marca de identidad" del constitucionalismo regional}

Conforme señalara en la sección anterior, resulta esencial para comprender lo ocurrido en materia constitucional en América Latina (1850-1890), prestarle atención a la exclusión o relegamiento de la "cuestión social". Fue explícita, durante el primer siglo del constitucionalismo regional, la pretensión de mantener limitados los derechos políticos. Se mantuvo al margen, por lo tanto, la atención de los problemas sociales ya presentes -vinculados, sobre todo, con las desigualdades económicas y políticas que el período colonial había alimentado y que el período post-colonial no había sabido resolver. El notable historiador argentino Tulio Halperín Dongi definió al programa que comenzó a primar a mediados del siglo XIX (y que él identifica con políticas como las promovidas por Alberdi), como un modelo de "autoritarismo progresista" -una "mezcla de rigor político y activismo económico".

La abierta resistencia impuesta por el liberalismo-conservador, frente a los primeros intentos serios de remediar la "cuestión social," adquirió una expresión dramática en la Convención Constituyente mexicana, de 1857.

El hecho es que dicha "resistencia constitucional" frente a lo social, vino de la mano de crecientes niveles de coerción y represión políticas que comenzaron a caracterizar a la región a finales del siglo XIX. El "estallido" de tales tensiones comenzaría a producirse a comienzos del siglo siguiente -un estallido que obligaría a la elite dirigente a dar inmediatas respuestas a las postergadas demandas sociales. Dichas respuestas, como sabemos, tomaron formas distintas y muy significativas que fueron desde el pasaje del "Estado Regulador" al "Estado de Bienestar", a la adopción de sistemas políticos más abiertos. A nivel constitucional, conforme veremos, la gran respuesta fue la aparición del constitucionalismo social, esto es, básicamente, la introducción de amplias listas de derechos sociales, políticos, culturales y económicos en la Constitución.

La gran ilustración de lo ocurrido entonces, a nivel constitucional, nos lo ofrece otra vez México, en donde la crisis adquirió niveles dramáticos que se expresaron, primero, en un exitoso movimiento revolucionario, y poco después, en una muy relevante reforma constitucional que tuvo como producto la Constitución de 1917. Se trató, según veremos, de la primer Constitución en el mundo que incluyó una larga lista de derechos sociales y económicos dentro de su cuerpo de artículos (Pisarello, 2011; Rabassa, 1991; Reyes Heroles, 1957).

Lo ocurrido en México en 1917, es de enorme relevancia para entender la historia constitucional mexicana y, más en general, la de toda la región. Sobre las reformas constitucionales promovidas entonces en México, quisiera destacar, al me- 
nos, dos cuestiones. Ante todo, el texto constitucional allí aprobado resulta todavía hoy venerado, a nivel nacional e internacional, por la radicalidad de su contenido. Sin embargo, prestando atención a la historia inmediata que lo precedió, podría decirse que el texto sorprende también por su moderación extrema, dado el contexto en el que apareciera: recordemos que, poco antes de que fuera aprobada la Constitución, la demanda común entre quienes la impulsaban comenzaba con la proclama "expropiación, confiscación, restitución" (Gilly, 1994). En segundo lugar, destacaría que una mirada más atenta de lo sucedido exige que distingamos entre los avances importantes que se lograran entonces, en términos constitucionales, y las continuidades que se mantuvieron con el pasado -lo que entonces no se hizo. Básicamente, si la Constitución de 1917 sorprende, es por el enorme cambio que impuso en materia de derechos; pero si ella genera cierta perplejidad, ello se debe al modo en que la Constitución se mantuvo fundamentalmente igual a sí misma, en todo lo relacionado con la estructura de poderes que estableció.

En todo caso, lo cierto es que en estos años aparece la segunda gran "marca de identidad" del constitucionalismo regional: las declaraciones de derechos robustas, extensas, generosas. Poco a poco, distintos países de la región fueron alumbrando declaraciones de derechos inspiradas en el ejemplo mexicano: Brasil en 1937, Bolivia en 1938, Cuba en 1940, Ecuador en 1945, Argentina en 1949 y Costa Rica en 1949, fueron algunos de ellos².

Habíamos visto, más arriba, de qué modo la consolidación (a mediados del siglo XIX) de una estructura de poderes concentrada, implicó dejar atrás el período de experimentación -en particular, con formas de organización del poder- propio de la primera mitad del siglo XIX, luego de la independencia. Vemos ahora, de qué modo, la afirmación de largas listas de derechos sociales, económicos y culturales (de mediados del siglo XX) vino a dejar atrás, también, el modelo de organización de los derechos que fuera dominante hasta entonces. El modelo que había prevalecido hasta entonces se caracterizaba por la incorporación de listas de derechos espartanas, breves y concentradas sobre lo que hoy denominamos "derechos liberales clásicos", relacionados con la propiedad, los contratos y algunas libertades básicas, como -típicamente- las de expresión, asociación, debido proceso y, ocasionalmente, la tolerancia religiosa. Se trataba de "libertades negativas", en la medida en que ellas requerían de parte del Estado un "no-hacer", relacionado con protecciones que se establecían en torno a los individuos: nadie interferiría con ninguno de ellos, afectándolos en su integridad física, sus libertades fundamentales, su propiedad.

Desde México 1917, Latinoamérica (y buena parte de Occidente) cambió, en términos constitucionales al menos. Las nuevas Constituciones creadas desde entonces vinieron a consagrar como propias las listas de derechos más complejas que rompían con el viejo molde y anexaban al esquema de derechos liberales vigentes los nuevos derechos sociales, económicos y culturales.

2 Por razones de espacio, paso por alto, por el momento, algunos intentos que se hicieron en la primera mitad del siglo, dirigidos a moderar en parte los poderes presidenciales. Me ocupo de ellos en Gargarella (2013). 


\section{El "último" período del constitucionalismo regional o el "nuevo constitucio- nalismo latinoamericano"}

A la luz de lo que hemos examinado hasta aquí, debiera resultar más sencillo reconocer por qué no corresponde hablar de la llegada de un "nuevo" tipo de constitucionalismo latinoamericano a partir de las últimas reformas aparecidas en la región. Lo que sostengo es, más bien, que el llamado "nuevo constitucionalismo" simplemente, refuerza algunos de los rasgos ya bien presentes en el marco constitucional de América Latina. Luego de la última oleada de reformas, nos encontramos con que: i) la parte orgánica de las nuevas constituciones sigue estando caracterizada por una estructura de poder concentrada políticamente y centralizada territorialmente; mientras que ii) la parte dogmática sigue distinguiéndose por la presencia de declaraciones de derechos robustas, generosas y extensas, que combinan derechos individuales y sociales de diverso tipo. En otros términos, la "doble marca" que comenzara a definir al constitucionalismo latinoamericano desde comienzos del siglo XX sigue tan vigente como entonces.

Decir lo anterior no implica afirmar que el "último" constitucionalismo latinoamericano ha hecho poco, o que no ha innovado suficientemente, o que no ha hecho nada importante. Simplemente señalo que las viejas estructuras se mantienen indemnes, lo cual es muy relevante, según entiendo, para reconocer el lugar en el que nos encontramos, y el tipo de problemas constitucionales que enfrentamos. Hago, a continuación, un breve repaso de lo que hoy nos ofrece el constitucionalismo latinoamericano, antes de detenerme en su análisis crítico.

\subsection{Declaraciones de Derechos}

Según entiendo, una característica fundamental de las "últimas" reformas estuvo dada por ciertas novedades que se introdujeron en las declaraciones de derechos. Tales novedades se dirigieron, al menos, a tres grandes objetivos tendientes a reparar tres grandes tipos de "faltas" históricas del constitucionalismo regional: i) su desatención a los derechos de "los postergados entre los postergados" (típicamente, comunidades indígenas); ii) su persistente desdén frente a los derechos humanos; y iii) su incapacidad para garantizar y hacer efectivos los derechos políticos de la mayoría de la participación, incentivando así una "ciudadanía activa". Veamos con algún detalle mayor lo señalado.

i) En primer lugar, en esta última etapa se expandieron los compromisos de tipo social asumidos por el constitucionalismo desde comienzos del siglo XX, para llegar así a sectores -fundamentalmente, los grupos indígenas, las mujeres- y cuestiones -los derechos de "tercera generación"- que no habían sido incluidas o reconocidas en aquella primera "oleada social" del constitucionalismo regional. De este modo, se le dio "ingreso" constitucional a los "excluidos entre los excluidos" -a los que no habían sido reconocidos por la Constitución, cuando se abrieron las puertas de la misma a la clase trabajadora. Las constituciones adquirieron así un perfil más "multicultural", y comenzaron a reconocer el valor de medidas tales como las accio- 
nes afirmativas. Asimismo, los constituyentes procuraron "actualizar" los viejos textos a partir de la "nueva conciencia de los derechos" (ambientales, de consumidores y usuarios, etc.) prevaleciente (ver, por ejemplo, Gargarella, Filippini y Cavana, 2011).

ii) En segundo lugar, las nuevas constituciones abrazaron de modo muy decisivo un compromiso con los derechos humanos. La causa constitucional de los derechos humanos es de enorme importancia porque expresa la reconciliación de partes significativas de la intelectualidad latinoamericana con las "listas de derechos" que habían despreciado durante décadas - muchas veces, por razones ideológicas vinculadas con resabios del viejo marxismo. Fundamentalmente, luego de la crudelísima oleada de dictaduras que azolaron a la región en los años 1970, comenzó a reconocerse en los ámbitos más diversos la necesidad de dar una protección amplia y efectiva a derechos humanos básicos. Se advirtió entonces que en figuras jurídicas tales como el debido proceso o la libertad de conciencia, estaba en juego directamente la vida -y no "meras cuestiones super-estructurales". Los modos en que los países latinoamericanos dieron cabida en sus constituciones a los derechos humanos fueron diversos, sobre todo a partir de la existencia de numerosos tratados de derechos humanos firmados por los distintos países de la región a lo largo de décadas. Habitualmente, se buscaron entonces fórmulas para reconocer el valor especial de aquellos tratados, a los que se dio estatus legal o supralegal. En la actualidad, otorgan estatus constitucional o supralegal a los tratados de derechos humanos: Argentina (artículo 75, inciso 22); Bolivia (artículo 256); Brasil (artículo 5); Colombia (artículo 93); Costa Rica (artículo 7); Ecuador (artículo 417); El Salvador (artículo 144); Guatemala (artículo 46); Honduras (artículo 18); Paraguay (artículo 141); Perú (artículo 56); República Dominicana (artículo 74); Venezuela (artículo 23). (Rossi y Filippini, 2010; Valadés, 2011).

iii) En tercer lugar, el último constitucionalismo latinoamericano procuró subsanar el grave déficit democrático que desde hace décadas parece afectar gravemente a la región. Estas reformas, entonces, se basaron en el generalizado reconocimiento de que las instituciones políticas funcionaban mal y no eran favorables a la intervención política de la ciudadanía (Dalmau, 2009; Viciano Pastor y Dalmau, 2011). Dado el carácter generalizado de tales reclamos de queja frente a la política, el constitucionalismo latinoamericano propuso fórmulas diversas, destinadas a abrir mayores oportunidades y espacios de control y decisión populares. En tal sentido, las últimas constituciones aprobadas en la región incluyen mecanismos de iniciativa popular (ver Gargarella, Filippini y Cavana, 2011).

Anticipándome a un análisis que haré más adelante, agregaría aquí los siguientes comentarios. En primer lugar, la tendencia a incorporar más y más derechos en la Constitución no resulta, obviamente, negativa. Ello, a pesar de la razonable objeción de que de ese modo se limita el alcance de la voluntad democrática de la ciudadanía. Podemos aceptar, en principio, el valor de que se afirmen ciertos intereses muy básicos de la ciudadanía y confiar en que dicho reconocimiento (constitucional) deje un margen suficiente para reflexiones y decisiones más apegadas a la coyuntura. En segundo lugar, podemos resistir, también, la razonable crítica, según la cual, no está bien generar tanta expectativa en nombre de "derechos nuevos": la Constitución 
bien puede representar, al menos en parte, un programa de acción política futura. En tal sentido, la afirmación de ciertos compromisos y ciertas aspiraciones puede resultar una combinación en principio aceptable: en parte, la Constitución es una apuesta a futuro (una apuesta a que las condiciones sociales que rodean a la Constitución -hoy tal vez desfavorables al florecimiento de todas sus cláusulas-, cambien en el futuro y ayuden a la realización de derechos que hoy parecen difíciles de poner en práctica).

Lo dicho, de todos modos, no niega, al menos, dos problemas que resultan evidentes desde un primer momento. Por un lado, la historia del constitucionalismo latinoamericano nos habla de una obcecación, por parte de los reformistas locales, por favorecer cambios sociales a través de la introducción de nuevos derechos (Couso, 2014; Gloppen, 2010; Salazar Ugarte, 2013; von Bogdandy et al., 2014; Wilson, 2010). Más precisamente, los constituyentes han demostrado una notable obsesión por la incorporación de más derechos, que no ha ido de la mano de una similar obsesión por mejorar -o cambiar, de modo acorde- la organización del poder (volveré luego sobre el punto). Tal cuestión nos llama la atención sobre un problema más vasto: que los constituyentes no han acompañado su pulsión recurrente por escribir más derechos en las constituciones, con una correlativa preocupación por las condiciones de posibilidad (materiales, políticas, legales) de los mismos derechos que afirmaron en los textos.

\subsection{Organización del Poder}

Sin mayor duda, podría decirse que la expansión de la lista de derechos operada por la última oleada de reformas en América Latina vino generalmente de la mano -si no fue directo producto- de la voluntad de facilitar procesos de reelección presidencial. Es decir, la voluntad reeleccionista de algunos presidentes motivó su disposición a intercambiar, en algunos casos, "más derechos" (ofrecidos como prenda a la oposición o a la ciudadanía) por "reelecciones". Casos como el de Carlos Menem en Argentina (y la Constitución de 1994), o el de Alberto Fujimori en Perú (y la Constitución de 1993) testimonian dichos acuerdos. Lamentablemente, de este modo, las reformas tendieron a quedar demasiado apegadas a cuestiones coyunturales, de momento. Ello, contra la idea alberdiana, más tradicional -y más venerable- de dedicar las escasas energías constitucionales a atender alguno de los "grandes dramas" sociales del momento (la lucha por la independencia o la lucha contra el atraso económico, en su momento; como hoy podríamos pensar en la lucha contra la desigualdad).

De todos modos, dada la extensión e importancia del período, tal vez convenga distinguir entre, al menos, dos etapas del mismo, vinculados con dos conjuntos de eventos de enorme importancia en toda la región. El primero, y ya mencionado, tiene que ver con la aparición de sangrientas dictaduras, en los años 1970. Y el segundo, referido a las crisis sociales que siguieron a la aplicación de "programas de ajuste estructural" ("neoliberales") en los años 1990. A muy grandes rasgos, podríamos decir que luego del grave período dictatorial, siguieron algunos intentos reformistas destinados a moderar, de alguna manera, los fuertes poderes remanentes en 
el Ejecutivo, y a confrontar algunos de los peores legados de la dictadura. Mientras tanto, luego del segundo período (el de la crisis social que siguió a los "ajustes económicos"), volvió a insistirse sobre la necesidad de concentrar el poder, a la vez que se buscó atender constitucionalmente algunas de las llagas abiertas por el desempleo y la desigualdad reinantes (Dalmau, 2009; Viciano Pastor y Dalmau, 2011).

Sobre la primera etapa, diría que durante algo más de una década (principalmente en los años 1980), varios países se orientaron a establecer límites en las reglas relativas a la reelección presidencial. Entre ellos, Ecuador (en 1978), Guatemala (1985), Honduras (1982), Colombia (1991) y Paraguay (1992). Aunque la tendencia a la moderación de los poderes presidenciales respondió a razones diversas, lo cierto es que la misma adquirió una dirección más definida (aunque, según veremos, en definitiva, breve) a partir de la citada sucesión de violentas dictaduras.

En efecto, y del mismo modo en que, en el área de los derechos, el pasaje de las dictaduras a democracias favoreció reformas constitucionales que incorporaron fuertes compromisos en materia de derechos humanos, en el área vinculada con la organización del poder, dicho período de oscurantismo político promovió la aparición de un inesperado consenso teórico orientado a limitar los poderes presidenciales. Por entonces, fueron muchos los juristas y cientistas sociales que comenzaron a cuestionar, no sólo al poder presidencial concentrado (que se había padecido de modo extremo durante las dictaduras), sino también a asociar-razonablemente- al llamado híper-presidencialismo latinoamericano, con la inestabilidad política y la recurrente práctica regional de los golpes de Estado. Al menos por un tiempo, la academia jurídica y política comenzó a sostener que la estabilidad podía tener muchas causas, pero que una de ellas era endógena al constitucionalismo y se vinculaba con los niveles de concentración de poder autorizados por las constituciones locales, que eran acompañados por una radical ausencia de "válvulas de escape" frente a las habituales crisis que afectaban a los regímenes de la región. El híper-presidencialismo latinoamericano, entonces, comenzó a ser identificado como factor clave en la explicación de los niveles de inestabilidad política que se habían registrado durante todo el siglo en la región (Nino, 1987; Nino, 1993; Nino, 1996; Linz y Stepan, 1978; Linz y Valenzuela, 1994).

Para Nino (1992, p, 38), por ejemplo, "el diagnóstico que se hizo es que un factor importante, aunque por cierto no el único, de la labilidad institucional argentina estuvo constituido por la formación de un presidencialismo hipertrofiado" a lo largo de la historia. El híper-presidencialismo implicaba concentrar poder y también responsabilidades y expectativas, en una sola persona, con mandato fijo durante años. Cualquier súbito desencanto con el presidente -cualquier crisis política o económica, cualquier quiebre en su salud, cualquier caída en su popularidad-tendía a traducirse entonces en una crisis del sistema político que carecía de válvulas de escape con las que remediar los desajustes, impidiendo la puesta en crisis de toda la estructura constitucional. Hubo un extendido acuerdo, entonces, según el cual la fuerte moderación o directa eliminación del sistema híper-presidencialista iba a permitir amortiguar las crisis, evitar su conversión en crisis sistémicas y atajar así la inestabilidad recurrente (Ackerman, 2000; Linz y Valenzuela, 1994). Sin embargo, lo cierto es que estos 
impulsos reformistas que apuntaban a moderar, de algún modo, al tradicional híperpresidencialismo latinoamericano, sólo fueron trasladados ocasional, y muy parcialmente, a la Constitución. Ocasionalmente, hubo limitaciones que se incluyeron en materia de poder presidencial y controles que se agregaron (Hartlyn, 2011; Hartlyn y Luna, 2007).

Conforme anticipáramos, por razones diversas -especialmente, por la mencionada crisis social desatada por los "programas de ajuste" que recorrieron la región en los años 1990-, el viejo temor al "caos" y la "anarquía" volvieron a hacer un llamado a la "necesidad" de un poder concentrado y una "autoridad fuerte", y derribaron, de un momento a otro, años de reflexión crítica y un creciente acuerdo sobre los males derivados del presidencialismo. Este tipo de cambios ayuda a explicar por qué, en materia de reelecciones presidenciales, la tendencia anti-reeleccionista que se había registrado en los años 1980 volvió a cambiar. Desde comienzos de los 1990, en particular, nueve casos sobre dieciséis de reformas constitucionales, nos refieren a países que reformaron sus textos fundamentales para facilitar la reelección presidencial (Negretto, 2013, p. 33). De este modo, las reformas comenzaron a orientarse más directamente a beneficiar a los actores que las promovían, favoreciendo la extensión de su mandato o la posibilidad pronta de su reingreso al poder.

Según un estudio reciente, los últimos cambios en materia reeleccionista pueden resumirse del siguiente modo: Argentina, en 1994, pasó de la no reelección inmediata a la reelección inmediata; Brasil, en 1997, hizo lo propio; Colombia, en 1991 prohibió la reelección inmediata, pero en el 2005 volvió a permitirla; en la República Dominica, en 1994, se prohibió la reelección inmediata, pero en el 2002 se la permitió; en Ecuador, en 1996, se pasó de la prohibición de la reelección a la permisión de la reelección no inmediata; en Nicaragua, en 1995, se pasó de la reelección inmediata a la no inmediata; en Panamá, en 1994, se determinó que la reelección sólo era permisible luego de dos términos presidenciales (en lugar de uno); en Paraguay, en 1992, la reelección quedó prohibida; en Perú, en 1993, se pasó de no inmediata a inmediata, pero en el año 2000 volvió a prohibirse la reelección inmediata; en Venezuela, en 1998, se pasó de la prohibición de reelección inmediata a su permisión (Payne et al., 2007).

Dejemos de lado, momentáneamente, la cuestión relacionada con la permisión o limitación de las posibilidades de la reelección presidencial, para prestar atención -de modo más general- a las diversas reformas introducidas en estas últimas décadas en materia de organización del poder. Ellas muestran facetas diversas que, en parte, responden a la misma lógica reeleccionista (concesión de controles a cambio de afirmación de los poderes presidenciales y/u obtención de derechos de reelección) $y$, en parte, a cansancios viejos con los modos tradicionales de ejercicio del poder. Resaltan, en tal sentido, varias reformas que habilitaron la elección popular de los alcaldes -Bolivia (en 1994); Colombia (1991); Paraguay (1992); Venezuela (1989). De modo similar, en Argentina, la reforma de 1994 consagró la elección popular del alcalde de la poderosa Ciudad de Buenos Aires, antes designado por el Ejecutivo Nacional. Por otro lado, hubo reformas electorales que tendieron a abrir la competición partidaria y a favorecer la aparición o el dominio de los sistemas de representación 
proporcional (Negretto, 2013, p. 25). En algunos casos, como el de México, las modificaciones electorales introducidas ayudaron a replantear significativamente las condiciones del juego político. Las modificaciones destinadas a moderar los poderes legislativos del Ejecutivo tuvieron alguna importancia (Brasil en 1988, Colombia en 1991, Paraguay en 1992, etc.), como aquellas que se dirigieron a fortalecer los poderes del Congreso, en relación con el Ejecutivo (el original mecanismo de "muerte cruzada" incorporada en Ecuador).

Importa mencionar, también, aquellas modificaciones dirigidas a fortalecer la independencia judicial y que pueden ser, asimismo, leídas como moderando los poderes del Ejecutivo y ayudando a incrementar los controles sobre el poder (RíosFigueroa, 2011). Se fortaleció o renovó la institución del Ministerio Público; aparecieron Consejos de la Magistratura destinados a transparentar el proceso de elección de jueces; se crearon cargos como el de Defensor del Pueblo; se favoreció, en los casos más extremos, la diversidad en la composición de los tribunales y, en algunos, se dejó la elección de jueces librada, en última instancia, a la decisión popular. En particular, destacaría aquellas reformas judiciales destinadas a favorecer el acceso del ciudadano común al litigio judicial -reformas, en el standing, o la legitimidad para accionar- como las de la tutela en Colombia (renovada de modo sustantivo a partir de la aparición de una nueva Corte Constitucional) o Costa Rica (donde se incorporara la afamada "Sala IV" en su tribunal superior, para atender cuestiones constitucionales) (Wilson, 2005; 2010), que implicaron modificaciones relevantes en el funcionamiento de la justicia (volveremos sobre el punto).

Aún cuando -en la mejor lectura- puede decirse que el constitucionalismo latinoamericano tomó ciertas medidas destinadas a limitar el poder presidencial, debe señalarse también que dichas medidas fueron en general muy modestas y que, habitualmente, quedaron cerca de la supervisión del propio Ejecutivo teóricamente afectado por las mismas. Más aún, tales tendencias contrastan, no sólo con las medidas en general tomadas en favor de las reelecciones presidenciales, sino también con otras que directamente vinieron a consagrar o expandir aún más la autoridad del Ejecutivo. En efecto, en algunas situaciones -como la de Argentina en 1994- las reformas certificaron constitucionalmente poderes que el Ejecutivo había ido acumulando de hecho y que la jurisprudencia le había ido reconociendo (i.e., expandiendo sus capacidades legislativas o su poder para actuar en situaciones de emergencia). En otras ocasiones, directamente, se asignaron al presidente capacidades para definir el presupuesto o involucrarse más directamente en la decisión de cuestiones económicas, para someter propuestas a la aprobación popular; etc. El punto más importante, de todas formas, tiene que ver con $e l$ modo en que se agregaron aquellas reformas dentro de la estructura existente y el lugar constitucional que se reservó para ellas dentro de una práctica (híper-presidencialista) ya consolidada (volveré sobre esta cuestión enseguida).

Según entiendo, en balance, estas reformas sobre la organización del poder han insistido con una matriz de poder político concentrado, que se ha consolidado como un elemento distintivo y definitorio del constitucionalismo regional ${ }^{3}$. Esta

3 Según el jurista colombiano Rodrigo Uprimny (2011, p. 10), las constituciones de la región "conservaron 
conclusión, por lo demás, es consistente con los datos que reporta el importante trabajo Comparative Constitutions Project y una serie de autores que han estado trabajado en el análisis comparativo de constituciones (Cheibub et al., 2011; Elkins et al., 2010 y comentarios al respecto en Hartlyn, 2011). En estos estudios se afirma que el desarrollo más destacado que ha mostrado el constitucionalismo regional se relaciona con el "incremento de cláusulas que conceden poderes legislativos a la rama Ejecutiva" (Cheibub et al., 2011, p. 1718), una evolución notable en torno a la cual todas las constituciones de la región han tendido a convergir (ibídem). Más fuertemente aún, dichos estudios afirman la existencia de un "modelo de poder presidencial propiamente latinoamericano, que incluye un poderoso papel del presidente en el área legislativa, y a la vez amplios poderes de emergencia" (ibidem, p. 1730).

\section{Describir lo ocurrido: reuniendo hilos}

En las páginas anteriores pudimos reconocer varias cuestiones importantes que nos ayudan a pensar mejor sobre los rasgos distintivos del constitucionalismo regional. Permítanme realizar un repaso sobre lo dicho.

En primer lugar, pudimos advertir que, a pesar de las más de doscientas reformas constitucionales producidas en doscientos años, el panorama que obtenemos no es el de un "caos constitucional" -cada nueva Constitución procurando instaurar un nuevo paradigma- sino un conjunto de constituciones que se han movido dentro de canales más bien estrechos, que finalmente se remontan a los grandes proyectos constitucionales que están en la historia más temprana del constitucionalismo regional (fundamentalmente, los relacionados con el Imperio Español -un proyecto de rasgos fundamentalmente conservadores-, la Revolución Norteamericana -un proyecto constitucional de rasgos típicamente liberales y la Revolución Francesa -un proyecto constitucional de rasgos radical-republicanos).

Particularmente, hemos señalado que en el constitucionalismo latinoamericano hubo un primer período de "experimentación" relativamente breve -al que Alberdi llamó el "primer derecho constitucional” de la región (1810-1850). En dicho período, las propuestas constitucionales que se pusieron en juego fueron más ambiciosas, imaginativas y diversas de lo que resultarían después. Luego de dicha etapa comenzaron a dejarse de lado muchas variables ensayadas en esos primeros años. Desde entonces, no sólo se abandonaron esas primeras búsquedas más "experimentales" -referidas a ensayos con instituciones novedosas y exóticas. De forma más general y relevante, el constitucionalismo latinoamericano dejó de oscilar entre sus tres proyectos "madre" -conservador, liberal, republicano- y comenzó a converger hacia canales más delgados, definidos -en su estructura organizativa principal- bajo el marco de un "liberalismo-conservador". En efecto, desde 1850 hasta hoy, podría decirse, el constitucionalismo ha tendido a moverse, en general (aunque no en todos

para el presidente poderes enormes frente al modelo presidencial clásico", aunque "hicieron esfuerzos por limitar el poder presidencial", a la vez que, de modo generalizado, tendieron a "aprobar la posibilidad de la reelección inmediata del presidente". Conviene leer también sobre el tema a Pisarello (2011, p. 193). 
los casos) dentro de carriles bastante angostos, que caracterizamos a partir de dos "marcas" principales.

La primera marca es la que dejó el acuerdo liberal-conservador de mediados del siglo XIX, vinculada con la organización del poder. Desde entonces, América Latina mantiene una división de poderes fundamentalmente tripartita, ladeada hacia el Poder Ejecutivo y territorialmente concentrada. Dicho esquema aparece basado, ante todo, en una general desconfianza hacia la ciudadanía - punto de encuentro que favorece decisivamente el acuerdo liberal-conservador-, lo cual ha redundado en sistemas políticos que desalientan (con las reservas conocidas) la participación autónoma de la ciudadanía y las diversas formas del control y decisión populares. Al mismo tiempo, el modelo liberal-conservador ha generado Poderes Legislativos con dificultades para funcionar autónomamente del Ejecutivo y Poderes Judiciales que aparecen habitualmente amenazados por la enorme capacidad de injerencia del partido dominante (expresado normalmente en el Ejecutivo) sobre sus propios asuntos.

La segunda marca, según vimos, fue la que quedó definida un siglo después, a mediados del siglo XX, cuando se integraron a la vieja estructura algunas de las demandas asociadas con los reclamos republicanos del siglo pasado, en nombre de la "cuestión social" ("cuestión social" que los líderes del pacto liberal-conservador habían decidido postergar). Desde entonces, se introdujeron cambios relevantes en las declaraciones de derechos propias del siglo XIX: hoy, las viejas listas de "derechos liberales clásicos" anexan amplios compromisos con derechos sociales, económicos y culturales.

Lo importante de lo que varió con la llegada del nuevo siglo, de todos modos, no se equipara con lo importante de lo que no cambió: permanecen desde el siglo XIX, casi intocadas, estructuras de poder a la vieja usanza, que consagran un poder concentrado y pocas posibilidades para la intervención popular en política.

Es en dicho marco que se produce la llegada del "nuevo" constitucionalismo regional. Aquí, otra vez, lo que predominan son las continuidades. En este caso -insistiría- continuidades gravemente acentuadas. Conforme viéramos, aquí no se producen cambios importantes, ni al nivel de la organización del poder, ni al nivel de las declaraciones de derechos. Las renovadas declaraciones de derechos se expanden aún más, para hacer mención a grupos antes no mencionados, a intereses antes no contemplados, o a derechos humanos antes dispersos u ocultos detrás de algunos de los derechos ya existentes. Simplemente, "no había mucho nuevo que inventar": los intereses fundamentales de la ciudadanía latinoamericana estaban básicamente contemplados ya en las viejas constituciones existentes.

No fue negativo, entonces, que se nombrara lo no nombrado (derechos nuevos, grupos particulares). Sin embargo, lo que se adoptó entonces fue, en todo caso, algo más o mejor de lo que ya se tenía, pero no algo estructuralmente distinto. Puede decirse algo similar en relación con los cambios introducidos al nivel de la organización del poder. Se produjeron algunas modificaciones interesantes, dentro de la organización tradicional, conforme viéramos: se acortaron mandatos a veces; se los extendieron poco después; se agregaron algunos controles nuevos sobre el Ejecutivo (Ministerio Público, Consejo de la Magistratura); a la vez que se le concedieron 
poderes que no se le habían reconocido antes (i.e., facultades de intervención en asuntos legislativos).

Tal vez, lo mejor que ocurrió en el área fue algo que sus creadores no anticiparon bien, pero que iba -aunque muy modestamente- en línea con lo que aquí venimos sugiriendo: cambios sobre alguna de las "palancas del poder", dirigidos a favorecer el acceso ciudadano a la "sala de máquinas" del constitucionalismo. Así, por ejemplo, en las "pequeñas pero significativas" variaciones impulsadas en materia judicial, y destinadas a facilitar y expandir la "legitimidad jurídica" necesaria para litigar judicialmente (Wilson, 2010). En todo caso, en sus rasgos más básicos, la vieja estructura de poderes se mantuvo cómoda con los cambios introducidos: las nuevas modificaciones parecían adaptarse bien al paladar de los viejos poderes prevalecientes. Paso entonces, más de lleno, al examen crítico de lo realizado en estos años.

\section{Evaluar lo escrito: modelos de democracia superpuestos y un constituciona- lismo que no termina de irse}

El recorrido hecho hasta aquí, fundamentalmente descriptivo, resulta -conforme entiendo- relevante, también, en términos normativos. No sólo por el hecho que la selección de datos responde a concepciones y entendimientos particulares, sino también porque ella tiene fuerza suficiente para ayudarnos a pensar sobre lo que se ha hecho y lo que no se ha hecho en materia constitucional en la región.

Entiendo que el análisis anterior nos ayuda a ir bastante más lejos de la primera idea que parece derivarse de lo dicho, según la cual lo nuevo es demasiado parecido a lo viejo. Por ello mismo, en esta sección, me adentraré algo más en la evaluación de lo acontecido para apoyar mejor la idea según la cual, la estructura que existe deja mucho que desear respecto de lo que ella misma proclama.

\subsection{Constituciones de "mezcla"}

Ante todo, destacaría que las constituciones latinoamericanas superponen modelos de democracia más bien opuestos, que se correlacionan con aspiraciones económicas, ideales políticos, compromisos legales -finalmente, modelos constitucionales- en tensión entre sí. Esta idea de "mezclar" pretensiones opuestas, superponiendo unas con otras, cuenta ya con buen arraigo en la tradición constitucional latinoamericana. Esa fue la manera principal en que se consolidó el acuerdo liberal-conservador. Fue muy habitual, según viéramos, que las aspiraciones propias del liberalismo se sumaran, sin mucho más, a las del conservadurismo. Entonces, se pudo agregar al esquema liberal de los "frenos y contrapesos" un Ejecutivo sobre-poderoso; como se pudo sumar, junto a las liberales declaraciones de tolerancia religiosa, otras que proclamaban el sesgo estatal a favor de la religión católica. Se escogió entonces un sistema de integración entre modelos -una mezcla constitucional- muy deficitaria, pudiendo haberse optado por otros sistemas de combinación más virtuosos. 
Para decirlo en otros términos más precisos, tratando de integrar las pretensiones opuestas propias de cualquier sociedad caracterizada por el "hecho del pluralismo" y el desacuerdo razonable, el sistema institucional puede reaccionar de formas diversas. Puede, por un lado, optar por propuestas de sintesis, tomando parte o lo mejor de cada propuesta, puede hacer un esfuerzo por combinar esas distintas pretensiones encontradas. Es lo que ocurre cuando -frente a demandas religiosas contradictorias- se afirma la imposibilidad de que cualquier religión sea impuesta por sobre las otras, permitiendo la coexistencia de todas. O se puede optar, en cambio, por -lo que consideraría indeseables- propuestas de acumulación, superponiendo sin más esas pretensiones encontradas. Es lo que hizo la Constitución argentina de 1853 (en buena medida todavía vigente) al afirmar, al mismo tiempo, la tolerancia de cultos (artículo $14 \mathrm{CN}$ ) y el status especial de la religión católica (artículo $2 \mathrm{CN}$ ). $\mathrm{O}$ es lo que hicieron la mayoría de las Constituciones latinoamericanas, al acumular simplemente las demandas de los conservadores a favor de un Ejecutivo fuerte, y las del liberalismo a favor de un sistema de equilibrios -dando lugar a un problemático híbrido latinoamericano que combina los "checks and balances" con un Ejecutivo súper-poderoso, capaz de desafiar ese equilibrio.

Las reformas que se sucedieron en la vida constitucional de la región, desde aquellos años fundacionales, parecen responder exactamente a la misma lógica de sus primeros ańos. La principal de las tensiones en conflicto aparecidas a partir de allí tiene que ver con la presencia de (con la acumulación de), al menos, modelos de democracia diferentes, orientados en direcciones opuestas. En efecto, en esa "doble marca" propia del constitucionalismo regional -poderes arreglados conforme a la regla dominante en el siglo XIX; derechos arreglados conforme a la regla dominante en el siglo XX- el constitucionalismo regional muestra su doble e inusual compromiso en materia democrática. Así, la estructura de poderes respondió -como responde aún- a valores democráticos propios del siglo XIX: baja participación popular, sectores excluidos, derechos políticos limitados - los mecanismos propios de la democracia censitaria. Mientras tanto, las nuevas declaraciones de derechos aparecen vinculadas con discursos y principios democráticos de "última generación". Se pretende una participación popular amplia, que busca apoyarse de diversas maneras: se abren oportunidades institucionales para que la ciudadanía gane capacidad de decisión y control (revocatorias de mandatos, etc.), se expanden los derechos políticos y, a la vez, se comprometen derechos sociales destinados a fortalecer todavía más el ingreso de las mayorías a la política (todo esto sujeto a limitaciones varias $)^{4}$. En términos democráticos, en definitiva, se afirma con una mano de la Constitución, lo que se niega con la otra.

Esa misma mezcla/acumulación problemática se da con otras cuestiones y en otros ámbitos de la Constitución: muchas de las nuevas constituciones (como las de Colombia o Perú, claramente) aparecen, a la vez, afirmando formulaciones económicas "neoliberales" y proclamas de fuerte contenido social que parecen indicar su vocación por formas económicas diferentes. Es muy común, también, en todas las

4 Es lo que, durante el siglo XIX, se expresaba en la tensión entre una idea de la política que pedía "libertades económicas" "abundantísimas" y "libertades políticas" limitadas; y otra que proponía libertades políticas amplias, y restricciones sobre las "libertades económicas" sin controles, entonces vigentes. 
constituciones "nuevas" comprometidas con los derechos indígenas, que se afirme simultáneamente el valor de la propiedad privada y el valor de la propiedad comunitaria (u otras similares), o que se afirme el valor de la economía privada, mixta, y pública, al mismo tiempo.

Podría decirse que este tipo de combinaciones resultan virtuosas: se trata, sobre todo, de un modo de comprometer, en el mismo proyecto constitucional, a formaciones políticas o grupos de interés en conflicto. Sin embargo, en términos constitucionales, este tipo de decisiones resultan cuestionables, por muchas razones y nos retrotraen a problemas a los que aludiéramos más arriba, al hablar del impacto intra-seccional de las reformas. Corresponde preguntarse, entonces: ¡cómo es que los nuevos derechos incorporados (i.e., derechos sociales, multiculturales, etc.) quedan vinculados con los derechos ya existentes? ¿Cómo se relaciona lo "nuevo" que incorporamos, con lo "viejo" que ya teníamos? Los problemas que se advierten son numerosos. Ante todo, a través de este tipo de decisiones el texto de la Constitución se torna confuso, menos claro. ¿De qué se trata, finalmente, la Constitución, cuando ella afirma, al mismo tiempo, pretensiones opuestas? De ese modo se abre la Constitución a interpretaciones contradictorias: ella pasa a decir mucho, nada o todo a la vez, en cuestiones fundamentales. ¿Cuál es el sentido, en tales casos, de tener una Constitución? Peor aún, así organizada, la Constitución induce a comportamientos equívocos y genera expectativas engañosas: tiene razón quien pasa a litigar en nombre de su propiedad, como quien pasa a impugnar dicha posesión en nombre de valores ancestrales. Por tomar un caso relevante: la introducción de "derechos de la naturaleza" ("suma kausay") en Constituciones como las de Ecuador o Bolivia, no sólo nos refiere a problemas más o menos evidentes (i.e., no es claro que se trate, como se alegara al adoptarse tales declaraciones, de "derechos ancestrales" de las comunidades indígenas - ¿cuáles?-; ni es claro que tenga sentido hablar de "derechos de la naturaleza"). Se puede valorar la intención de incorporar "principios interpretativos" nuevos, diferentes a los tradicionales (Acosta, 2008); sin embargo, es difícil no preguntarse cómo entender tales principios cuando la Constitución no reniega de otros principios e instituciones contrarias (i.e., vinculadas con tradicionales derechos de propiedad).

Encontramos problemas similares a los que reconocemos en relación con los "viejos" y "nuevos" derechos en la Constitución, en el vínculo que se da entre las "viejas" estructuras de poder y las "nuevas" instituciones que se incorporan. Una buena ilustración de lo dicho puede encontrarse en el ejemplo del llamado "choque de trenes" en Colombia, que enfrentó a la vieja Corte Suprema Colombiana, con la nueva Corte Constitucional introducida por la Constitución de 1991. Ambas instituciones mantienen desde hace años una relación de rivalidad y tensión que comenzó con el mismo nacimiento de la última y que implica persistentes disputas de poder, y una nociva competencia entre las dos Cortes (Cepeda, 2004; Uprimny, 2011). Otro ejemplo relevante en la materia puede encontrarse en Argentina, cuando examinamos las relaciones entre la Corte Suprema (presente desde la primera Constitución, de 1853) y el Consejo de la Magistratura, que fuera introducido por la reforma constitucional de 1994. Aquí vemos otra vez relaciones de fuerte tensión 
entre los dos organismos (tensiones que podían anticiparse al momento de la creación del Consejo) relacionadas con la dificultad de definir con exactitud el área de la competencia exclusiva de cada una de las instituciones, pero también (y a partir de allí) con un cierto hostigamiento de la Corte sobre el Consejo, donde la primera aparece celosa de perder facultades que considera propias (Gargarella, 1996).

En situaciones como las descriptas, encontramos una falta de reflexión, o reflexión impropia por parte de los constituyentes latinoamericanos. Por hipocresía, demagogia, descuido o algún malentendido, aquellos actúan mal cuando no cuidan los modos en que "el pasado" va a relacionarse con "el presente" -la "vieja Constitución" va a recibir a los aspectos "nuevos" que se le incorporen.

\subsection{Las viejas estructuras contra los nuevos derechos}

Lo dicho hasta aquí está especialmente vinculado con lo que llamáramos el impacto intra-seccional de las reformas. Pero los problemas en cuestión se extienden también -y se agravan- en lo relacionado con lo que denomináramos el impacto inter-seccional de las reformas. Me refiero a los modos en que la incorporación de nuevos derechos afecta la organización del poder, o los modos en que lo que hagamos o dejemos de hacer en la organización del poder afectan a las declaraciones de derechos.

Los problemas que aparecen entonces se producen en diferentes niveles, y quisiera mencionar a algunos de ellos, aún cuando no pueda detenerme tanto como desearía en el análisis de los mismos. La dificultad general que enmarca a todas las restantes es ya clásica en el constitucionalismo, y refiere a las tensiones existentes entre Constitución y democracia (Elster y Slagstad, 1993). En principio, suele decirse, existe -algo así como- una relación de "suma cero" entre ambas esferas. Así, la incorporación de más y más derechos en la Constitución sugiere un menor espacio para la deliberación colectiva: cuantas más preguntas se resuelven de antemano a través de la afirmación de derechos ("cartas de triunfo" frente a las pretensiones democráticas, como sostuviera Ronald Dworkin, 1977), menos espacio le queda a la ciudadanía democrática para decidir conforme a sus necesidades y pretensiones presentes.

De modo más específico, aparece la cuestión acerca de cómo "transferimos poder," dentro de la Constitución, a través de cada modificación que le incorporamos. Como ejemplo podemos citar el siguiente hecho: la deseada incorporación de derechos sociales (promovida, habitualmente, por reformistas democráticos) importe la transferencia de poderes adicionales al Poder Judicial (esto es, la rama menos democrática del poder). Cabe señalar que problemas como el citado resultan de especial relevancia en el constitucionalismo contemporáneo, dado que muchos de los defensores de los derechos sociales tienen como propósito reforzar el "poder popular" antes que el poder de las jerarquías legales existentes. Y, sin embargo, al actuar como actúan, generan un "impacto constitucional" en parte opuesto al que dicen buscar.

Finalmente, llegamos a la cuestión que aquí más me interesa y preocupa, que es la relacionada con los modos en que las "viejas estructuras" bloquean las "nuevas propuestas", o tornan difícil la implementación de las mismas -típicamente, en este caso, el modo en que la vieja organización del poder obstaculiza la realización de 
los nuevos derechos sociales y multiculturales. El problema en juego no nos refiere, meramente, a una cuestión de "simple descuido" en la redacción constitucional (digamos, introducimos nuevos derechos, sin prestarle atención a los modos en que reacciona o va a reaccionar la vieja organización del poder). Se trata, ante todo, de que no reconocemos el peculiar lugar que ocupa la parte "orgánica" no reformada: lo que está en juego es el núcleo básico de la organización de poderes, esto es, la sala de máquinas de la Constitución. Por supuesto, puede entenderse que existan dificultades para reconocer todos los cambios que es necesario agregar, para "darle vida efectiva" a la modificación constitucional que estamos más interesados en incorporar. Sin embargo, dejar de lado, directamente, la pregunta acerca de cómo va a responder la "sala de máquinas" constitucional frente a los demás cambios constitucionales que introduzcamos ("más derechos"), es dejar de lado lo más importante. En aquella "sala de máquinas" reside, justamente, el corazón de la Constitución: no puede operarse sobre la Constitución dándole la espalda al modo en que la organización del poder reacciona (o, previsiblemente, va a reaccionar) frente a las modificaciones que le introducimos.

\section{Bibliografía}

Acosta, A. (2008). "El Buen Vivir: una oportunidad para construir". Ecuador Debate, 75, pp. 33-48.

Ackerman, Bruce (2000). “The New Separation of Powers", Harvard Law Review, January 113 (3), pp. 633-729.

Alberdi, Juan Bautista (1920). Obras Selectas. Edición de Joaquín V. González. Buenos Aires: La Facultad. Alberdi, Juan Bautista (1981). Bases y puntos de partida para la organización política de la República Argentina. Buenos Aires: Plus Ultra.

Barros Arana, Diego (1913). Un decenio de la historia de Chile. Tomos I y II. Santiago de Chile: Imprenta Universitaria.

Basadre, Jorge (1949). Historia de la República del Perú. Lima: Editorial Cultura Antártica.

Bauzá, Francisco (1887). Estudios constitucionales, Montevideo: Establecimiento tipográfico-editorial de la Librería Nacional.

Bazant, Jan (1977). A Concise History of México. From Hidalgo to Cárdenas, 1805-1940. Cambridge: Cambridge University Press.

Blanco Acevedo, Pablo (1939). Estudios constitucionales. Montevideo.

Bushnell, David (1993). The Making of Modern Colombia. A Nation in Spite of Itself. Berkeley: University of California Press.

Carpizo, J. (2009). “Tendencias actuales del constitucionalismo latinoamericano”. Disponible en:

http://www.pj.gov.py/ebook/libros_files/Ponencia_Jorge_Carpizo_Tendencias_Const.pdf [Acceso: marzo de 2018].

Cepeda, Manuel (2004). "Judicial Activism in a Violent Context: The Origin, Role, and Impact of the Colombian Constitutional Court”, 3 (529) Washington University Global Studies Law Review.

Cheibub, J. C.; Elkins, Z.; Ginsburg, T. (2011). "Latin American Presidentialism in Comparative and Historical Perspective”, Texas Law Review, 89 (7), pp. 1701-1741.

Couso, J. (2014). "Las democracias radicales y el nuevo constitucionalismo latinoamericano", Derechos humanos: posibilidades teóricas y desafíos prácticos, SELA. Buenos Aires: Libraria. 
Dworkin, Ronald (1977). Taking Rights Seriously. Cambridge: Harvard University Press.

Elkins, Z.; Ginsburg, T. y Melton, J. (2010). “The Comparative Constitutions Project: A Cross-National Historical Database of Written Constitutions”. Disponible en: http://www.comparativeconstitutionsproject.org [Acceso: marzo de 2018].

Elster, Jon y Slagstad, Rune (Editores) (1993). Constitutionalism and Democracy. Cambridge: Cambridge University Press.

Gargarella, Roberto (1996). La justicia frente al gobierno. Barcelona: Ariel.

Gargarella, Roberto (2010). The Legal Foundations of Inequality. Cambridge: Cambridge University Press.

Gargarella, Roberto (2013). Latin American Constitutionalism, 1810-2010. Oxford: Oxford University Press.

Gargarella R., Filippini L. y Cavana, A. (2011). Recientes reformas constitucionales en América Latina, Reporte UNDP.

Gilly, Adolfo (1994). La revolución interrumpida. México: Ediciones Era.

Gloppen, S., Wilson, B., Gargarella, R., Skaar, E. y Kinander, M. (2010). Courts and Power in Latin America and Africa. New York: Palgrave.

Halperín Donghi, Tulio (1980). Proyecto y Construcción de una Nación. Caracas: Biblioteca Ayacucho.

Hartlyn, J. (2011). “Commentary: Constitutional Structure in Latin America”, Texas Law Review, 89 (7), pp. 1977-1983.

Hartlyn, J.; Luna, J. P. (2007). "Constitutional Reform in Latin America: Intentions and Outcomes", trabajo presentado en 27o. Congreso de la Asociación de Estudios Latinomericanos, LASA Montreal, Canadá.

Jaksic, Iván (2001). Andrés Bello. La pasión por el orden. Santiago de Chile: Editorial Universitaria.

Jaramillo Uribe, J. (1964). El pensamiento colombiano en el siglo XIX. Bogotá: Editorial Temis.

Linz, Juan y Stepan, Alfred (1978). The Breakdown of Democratic Regimes. Baltimore: The John Hopkins University Press.

Linz, Juan y Valenzuela, Arturo (1994). The Failure of Presidential Democracy, Baltimore: The John Hopkins University Press.

Loveman, Brian (1993). The Constitution of Tyranny. Regimes of Exception in Spanish America. Pittsburg: University of Pittsburg Press.

Martínez Dalmau, R. (2009). ¿Qué es el 'nuevo constitucionalismo latinoamericano’?”, Gaceta Constitucional, n. 52, Lima.

Molina, Gerardo (1973). Las Ideas Liberales en Colombia, 1849-1914. Bogotá: Colección Manuales Universitarios, Tercer Mundo.

Negretto, Gabriel (2013). Making Constitutions: Presidents, Parties, and Institutional Choice in Latin America. Cambridge: Cambridge University Press.

Negretto, G. y Aguilar Rivera, J. A. (2000). "Liberalism and Emergency Powers in Latin America: Reflections on Carl Schmitt and the Theory of Constitutional Dictatorship", Cardozo Law Review, 21 (5-6), pp. 1797-1823.

Nino, Carlos (ed.) (1987). Presidencialismo vs. Parlamentarismo. Buenos Aires: Consejo para la Consolidación de la Democracia.

Nino, C. (1992). “¿Qué reforma constitucional?”, Propuesta y control, vol. 21, pp. 37-59.

Nino, C. (1993). "Transition to Democracy, Corporatism and Presidentialism with Special Reference to Latin America”, en D. Greenberg, S. Katz, B. Oliveiro y S. Wheatley (eds.): Constitutionalism and Democracy, Transitions in the Contemporary World. Oxford: Oxford University Press. 
Nino, C. (1996). "Hyperpresidentialism and Constitutional Reform in Argentina", en A. Liphart A. y C. Waisman, eds.: Institutional Design in New Democracies, New York: Westview Press.

Payne, M., Zovatto, D. y Mateo Díaz, M. (2007). Democracies in Development: Politics and Reform in Latin America. Washington DC: Inter American Development Bank.

Pisarello, Gerardo (2011). Un largo termidor. La ofensiva del constitucionalismo antidemocrático. Madrid: Trotta.

Rabassa, E. (1991). “Historia de las Constituciones mexicanas”, en J. L. Soberanes Fernández. El Derecho en México. Una visión de conjunto, Tomo I. México: Universidad Nacional Autónoma de México.

Reyes Heroles, Jesús (1957). El liberalismo mexicano. México: Universidad Nacional de México.

Ríos-Figueroa, J. (2011). "Institutions for Constitutional Justice in Latin America”, en G. Helmke y J. Ríos-Figueroa (eds.): Courts in Latin America. New York: Cambridge University Press.

Rodríguez Garavito, César (2011). El derecho en América Latina. Buenos Aires: Siglo XXI.

Rossi, J. y Filippini, L. (2010). "El derecho internacional en la justiciabilidad de los derechos sociales El caso de Latinoamérica”, en P. Arcidiácono et al.: Derechos sociales: justicia, política y economía en América Latina. Bogotá: Siglo del Hombre, Universidad de Los Andes.

Salazar Ugarte, P. (2013). "El nuevo constitucionalismo latinoamericano (una perspectiva crítica)", en L. González Pérez, D. Valadés (comps.): Constitucionalismo contemporáneo. Homenaje a Jorge Carpizo. México. Disponible en:

http://biblio.juridicas.unam.mx/libros/libro.htm?l=3271 [Acceso: marzo de 2018].

Samper, José María (1861). Ensayo sobre las revoluciones políticas y la condición social de las repúblicas colombianas. París: Imprenta de E. Thunot.

Unger, R. (1987). "El sistema de gobierno que le conviene a Brasil”, en Presidencialismo vs. Parlamentarismo. Buenos Aires: Consejo para la Consolidación de la Democracia.

Uprimny, R. (2011). "The Recent Transformation of Constitutional Law in Latin America: Trends and Challenges”, Texas Law Review, vol. 89 (7), pp. 1587-1610.

Valadés, D., et al. (eds.) (2011). Ideas e instituciones constitucionales en el siglo XX. México: Siglo XXI.

Vallenilla Lanz, Laureano (1919). Cesarismo democrático. Estudios sobre las bases sociológicas de la Constitución de Venezuela. Caracas

Viciano Pastor, R. y Martínez Dalmau, R. (2011). “Fundamentos teóricos y prácticos del nuevo constitucionalismo latinoamericano", Gaceta Constitucional n. 48, Lima, pp. 307-328.

von Bogdandy, A., Fix Fierro, H., Morales Antoniazzi, M. (coords.) (2014). Ius Constitutionale Commune en América Latina. Rasgos, Potencialidades y Desafíos. México, UNAM.

Wilson, B. (2010), "Explaining the Rise of Accountability Functions of Costa Rica's Constitutional Court," en S. Gloppen et al.: Courts and Power in Latin America and Africa. New York: Palgrave, 63-82. 